\title{
A Parallel Environment Designing for OWL Thinking
}

\author{
Ms. Sanjana C Madargi ${ }^{1,3, *}$ and Dr. Leena R Ragha ${ }^{2}$ Mrs. Vanita $M$ Mane $^{3}$ \\ ${ }^{1}$ Department of Computer Engineering R.A.I.T Nerul, Navi Mumbai, 400706. \\ ${ }^{2}$ Department of Computer Engineering R.A.I.T Nerul, Navi Mumbai, 400706 \\ ${ }^{3}$ Department of Computer Engineering R.A.I.T Nerul, Navi Mumbai, 400706
}

\begin{abstract}
A huge volume of information available today is in the form of images and searching the wanted images is very difficult and highly time-consuming. The search may take longer periods as the search volume on the internet is very huge and also the relevance of extracted images is still not up to the mark. The technologies like ontology and languages like OWL help us to tag the images that describe the semantic of the images. Hence, it helps in faster searching of the wanted images. Also, another challenge with OWL and Semantic web is the speed in which one can derive the relationships between various objects extracted from the images. The challenge is to extract the semantic from the images more efficiently using a parallel approach. In this paper, we explore the different techniques for generating semantic knowledge using parallel approaches like the T-box approach, merge classification, extract concept for matching ontology. We propose an enhanced method to speed-up the computation by combining T-box and merge classification techniques.
\end{abstract}

Keywords: OWL, Multiple-threading, Merge sort, Ontology.

\section{Introduction}

With technology growth, everything is getting automated these days at a faster pace. Human adopts to the technologies that give him the best results. Nowadays we communicate with each other on the web. Thus, a lot of data is generated in the form of text and images. As a human, it is quite simple to understand the natural language but it is a challenging task for a system, thus natural language processing is required. A picture worth a thousand words. And hence, communication through images has taken over the world. There is a need for categorization of these images for faster searching from the huge volume of various categories and domains of images. Traditional co-ordination frameworks have essentially focused on syntactic organization and concentrated less on the semantic information. Given the dynamic and complex environment, where data from the scope of heterogeneous spaces is traded, it is critical to think about the natural significance of data.

The understanding of ontology procedure requires a detailed examination of ideas, terms, and connections in different areas of interest. It requests a minute exchange of information related to ideas since it is conveyed for an away from the area. Ontology learning is beginning to develop as a sub-area of ontology designing because of the fast increment of web archives and the propelled methods shared by the data recovery, AI, regular language preparation, and artificial intelligence networks.

*e-mail: sanjanacmadargi@gmail.com
Philosophy portrays an area, while an information base (given ontology) depicts a specific situation. The conceptualization portrays information about the area in the form of objects and not about the specific situation in the space. Also, the conceptualization does not change or changes once in a while. Philosophy is then the determination of this conceptualization and is indicated by utilizing a specific display of dialect and specific terms. Formal determination is required to have the capacity to process ontology and work on object identification simultaneously.

Therefore, looking through the immense volume of data as pictures extracting the needed pictures that too in real-time is a challenge and we propose to work on this front. The paper explores the parallel technologies used by different researchers in this domain of image information retrieval in section 2 as part of a literature survey. Section 3 concentrates on the proposed method and applies it to an example to demonstrate how it works. The practical implementation of it in the distributed environment and the time complexity analysis is beyond the scope of the paper.

\section{Literature Review}

We surveyed research work based on parallel computing techniques and algorithms. The overall methodology applied by different researchers is explained below:

Gauthami Latha [1] et.al. the author explain about semantic-based inquiry gives suitable and applicable outcomes than those of conventional watchword based hunt. The effectiveness of the semantic-put together pursuit depends with respect to utilizing the properties and connections among the pictures are named "Ontology" and it is built by thinking about the degree of human 
comprehension. Ontology is developed by utilizing the low-level highlights like surface, shading, and state of pictures which duplicates human comprehension. In this way, philosophy is viewed as progressively helpful for the recovery of pictures that are semantic-based. The picture recovery strategy named Content-based low-level highlights of the picture than the whole picture. The other option for picture recovery is supposed to be proficient as it considers low-level highlights of the picture than the whole picture. Another option for the picture recovery process is performed utilizing philosophy based portrayals named Ontology-based picture recovery which utilized the information portrayals that incorporate the setting of substance based picture recovery with the content-based picture recovery highlights. The key thought of philosophy is to introduce the picture in semantic documentation which is interpret able for the machine prompting the effective recovery of results. The Ontology gives the portrayal of information utilizing the elements indicating the OWL with the development of classes, relations, characteristics and occasions among area people which is upheld consistently as Rules.

Zixi Quan et.al. [2, 3] the author described novel parallel OWL classification architecture. They have proposed a random division strategy for good speed- up and scalable for ontology's of large sizes and in end group division strategy to complete T-box classification. In this paper, it is compared with sequential classification methods and limitations of parallel approaches. It deals with extensive scale ontology to show signs of improvement execution contrasted with existing consecutive methods. The plan is to accomplish a superior load balance answer for the two parallel phrases. By parallel T-box classification, technique demonstrates promising outcomes that influence to trust it could be connected to ontology's with a lot bigger size and confounded ontology with promising run time and a superior speed-up. Parallel T-box classification approach, in which it concentrates on parallelism on basis of threading. By multiple threads co-exists and based on randomizing distribution on a given data set. For example, an image is given in which describes fruit, vehicle, chocolate, and animal. To classify that which set will give the exact match with refined meaning. Consider some sets of threads present splitting sub-parts as $\mathrm{P}, \mathrm{Q}, \mathrm{R}, \mathrm{S}$ for testing for the similarities. And assume the categories as C1 as Fruits, C2 as Cars, C3 as Chocolate and $\mathrm{C} 4$ as Dogs.

Step 1: Consider the below database of the objects as shown:

$S_{P}=\{$ Apple, SUV $\}$

$S_{Q}=\{$ Orange, Cadbury, Peach, Sedan $\}$

$S_{R}=\{$ Mango, i20, Audi, Snickers, Poodle, Pug $\}$

$S_{S}=\{$ Honda city, German shepherd, Banana, Kit-kat, Water Melon\}.

Step 2: As per the above given categories for each data which is fetched from the dictionary, groups will be generated as given below:

Fruit: Group 1: $C_{1}=$ \{Apple, Orange, Peach, Mango,
Banana, Water Melon $\}$

Car: Group 2: $C_{2}=\{$ SUV, Audi, i20, Honda city, Sedan $\}$

Chocolate: Group 3: $C_{3}=$ \{Cadbury, Kit-kat, Snickers $\}$

Dog: Group 4: $C_{4}=\{$ German shepherd, Poodle, Pug $\}$.

Step 3: Four groups $\left\{t t_{1}, t t_{2}, t t_{3}, t t_{4}\right\}$ are created from above categorization as below:

$t t_{1}:\{1,2\}$

$t t_{2}:\{1,3,1,2\}$

$t_{3}:\{1,2,2,3,4,4\}$

$t t_{4}:\{2,4,2,1,3,2\}$.

Step 4: Finally, suppose $S_{X}=\{$ Apple, Sedan, Pug $\}$. The possible sets from above sets are: $t t_{1}=\{1\}, t t_{2}=\{1,2\}$, $t t_{3}=\{1,3\}, t t_{4}=\{1,4\}, t t_{5}=\{1,2,3\}, t t_{6}=\{1,2,4\}, t t_{7}=$ $\{1,3,4\}, t t_{8}=\{1,2,3,4\}$. The result of image is group $6\left(t t_{6}\right)$ contains the set of $\{1,2,4\}$ which match with $\{$ Apple, Sedan, Pug\}.

Kejia Wu et.al. [4] provides exploration is motivated by the ubiquitous accessibility of multiprocessor PCs and the perception that accessible Web Ontology Language (OWL) reasoners just make utilization of a solitary processor. This much work has only researched the performance of the concurrent merge classification. Parallel merge classification uses merge sort, data set will be divided into $\mathrm{n}$ different parts, each part sorted and operated on a given part of data by a particular processing unit.

Mina Aslani et.al. [5] described an architecture for parallelism well-known algorithm for T-box classification. Work was focused on ontology's where free partitions cannot be effortlessly built; in this way did not utilize the recently referenced approaches in the system. Also plan to implement and test approach in a multi-center and multiprocessor condition.

Mina Aslani et.al. [6] one of the most frequently used inference services of description logic of reasoners is the classification of T-boxes with a subsumption pecking order of all named ideas as the outcome.

Markus Fronk et.al. [7] introduced a path to explaining services utilizing OWL- DL standard reasoners. The solution described in this paper especially versatile for the robotizing of the revelation and the arrangement of web services.

Alaa Qassim et.al. [8] this paper gives a depiction of the plan of the automatic annotation system. The design phase of any exploration is the most significant part as it depicts how the framework will be organized to meet the prerequisites. Matching ontology on the semantic web of documents briefly and expressly through separating just the related resultant semantics from the document. For example, Mississippi the state, from Mississippi the stream, narrow searching of the word from the dictionary which defines the meaning recognize the various words. It can resolve the ambiguity of all the contexts on which it is referred.

Andreas Steigmiller et.al. [9] presents Konclude, a high performance reasoned for the description logic SROIQV. The supported ontology language is a super-set of the logic fundamental OWL 2 reached out by nominal schemas, which takes into consideration communicating discretionary DL-safe standards. Konclude's think- 
ing center is fundamentally founded on the well-known tableau calculus for expressive description logic. This paper depicts Konclude's interface choices, reasoner design, preparing the work process, and key improvements.

We have studied the techniques which are related to the computation work and semantics related to OWL ontology and semantics of image too. The precision of ontology classification in this context is that semantic labeling helps in arranging the general pictures, for quicker inquiry and specific individual's pictures can be understood deeply. The parallel T-box classification contains several subsumption sets due to which the performance of the overall classification of ontology gets lowered and parallel merge classification is applied to the larger samples will consume more amount of time. So, we propose to address these issues in the proposed method section below with some data-set to understand it.

\section{Proposed Method}

The proposed solution aims to overcome the individual drawbacks of parallel T- box and merge classification approaches. The strategy is to mix various threading and consolidation sort technique. The benefits compared to sequential execution with parallelization is that the task can be done concurrently with interaction on similar processors between them which leads to time-saving and easily share data-sets.

Working on the proposed technique and its flow is shown in Figure 1. From examples, we obtained constraints of individual techniques Parallel T-box classification approach and parallel merge classification. The proposed technique defeats the confinement of calculation. In merge-sort, multiple threading is used where each part would be operated simultaneously by a particular thread. The basic idea is to produce multiple threads that may exploit a system with a single processor with multiple cores or a multi-processor system. In a single processor system with multi-threading, context switching would be faster, the thread can easily share their data sets and stay away from conceivable race conditions for updates of shared information. Merge sort algorithm helps in better context switching and threads can easily share data by dividing and sorting. Hence improving the efficiency of computation can be achieved. For parallel computing, we require a multi-core processor with OpenMP or MPI techniques to support computing.

The steps followed are as follows:

Step 1: Loading the text or images and content is stored in the distributed memory so that work can be done at a concurrent time. As to fetch information in detail have dictionary-engine in which it has two parts. First is a tag and second are tittles which will be pointing to the keywords, so that feature extraction becomes easier.

Step 2: Dividing tasks into equal numbers that are waiting to get completed their jobs in the queue.

Step 3: Categorize the components according to who comes before the execution time slice. As the number of tasks is waiting for their execution, this helps to take

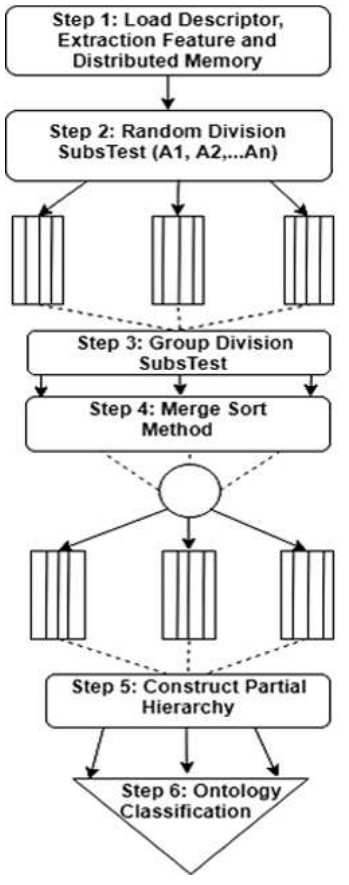

Figure 1. Generalized Working of Proposed Method

concurrent work and to improve the speed-up factor.

Step 4: This step is where the merge sort is to be applied. It takes the sets from the above step, divides the sets by $n 1$ number of tasks of the same type, from that divide the sets by $n 2$ number of sub-tasks for the total number of tasks until we get the last sub-parts separately.

Step 5: After the task is divided, fragmented order for the same sets of data will be constructed and stored to create an ontology.

Step 6: Finally, the ontology will be classified which narrows down the search process. Here we achieve the feasible solution with the finer meaning of the individual identified objects.

The whole process is demonstrated using data-set to conclude about the given proposed method the following example.

Example: The image will have objects that describe the lifestyle of a person. It is required to identify the different objects and group them to label the image in the end w.r.t. the lifestyle of a person. Here we assume that the objects are extracted from the image and are associated with object names. Also, let us assume the universal dictionary has the following groups.

Fruit: Group 1: G1 = \{Apple, Orange, Peach, Mango, Banana, Water Melon $\}$

Car: Group 2: G2 = \{SUV, Audi, i20, Honda city, Sedan $\}$ Scenery: Group 3: G3 = \{Cloudy Sky, Sleepy, Snow, Sports ground, Forest $\}$

Step 1: Divide the given sets randomly as the task is dispatched and then merge them to form groups based on the predefined Categories they belong to in the dictionary database. 
Step 2: Form the local group of sets with similar context as of dictionary.

$\mathrm{C} 1=$ Fruit $=$ Banana, Apple, Mango,

$\mathrm{C} 2=\mathrm{Car}=\mathrm{SUV}$, Audi, i20,

C3 = Scenery $=$ Cloudy Sky, Sleepy, Snow, Sports ground, Forest.

Step 3: Merge the above groups based on the relations we want between the different sets using the categorized words to form a tree as shown in Figure 2.

Step 4: In this step, Hierarchy is constructed with the help of the above tree and the lifestyle which is person-related can be further divided into with other entities like rich, poor, lavish, religious, balanced, sportsman, trekker, etc and again it gets divided accordingly to get the semantic ontology for the given image.

Step 5: Ontology of the images thus extracted is classified with this context-based interpretation.

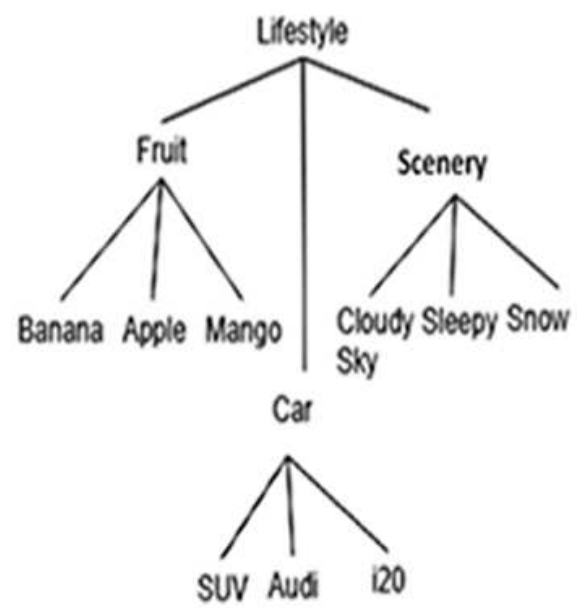

Figure 2. Result produced in Step 4

\section{Results and conclusions}

This kind of semantic tagging helps in categorizing the general images, for faster search. It also helps to track a particular person's images to extract the lifestyle of the person.

For an input image with objects like a person with SUV, Apple, and Snow the result will be a trekker.
One can also go beyond the lifestyle to identify his spending habits and economic status. Thus, the tree starts becoming deeper in levels. Based on the expressions given in their evaluation by the reasoning of a standard reasoner and behavioral aspects of a web application service, the request can be automatically executed to generate the semantics.

\section{References}

[1] Gauthami Latha, Ch. Satyanarayana, Y. Srinivas.: Semantic Image Annotation using Ontology And SPARQL. Web Semantics: Science, Services and Agents on the World Wide Web 2728, 2014. International Journal of Innovative Technology and Exploring Engineering (IJITEE) ISSN: 2278-3075, Volume-9 Issue-3, January 2020.

[2] Zixi Quan, Volker Haarslev.: A parallel computing architecture for high-performance OWL reasoning. Parallel Computing, 2018.

[3] Z Quan, V.Haarslev.: A Parallel Shared-Memory Architecture for OWL Ontology Classification. 46th International Conference on Parallel Processing Workshops, IEEE, 2017.

[4] Kejia Wu, V. Haarslev.: Parallel OWL Reasoning: Merge Classification. JIST, Revised Selected Papers of the Third Joint International Conference on Semantic Technology, 2013.

[5] Mina Aslani, V. Haarslev.: Parallel T-box Classification in Description Logic's First Experimental Results. Proceedings of the 2010 conference on ECAI: 19th European Conference on Artificial Intelligence, 2010.

[6] Mina Aslani, V. Haarslev.: Towards Parallel Classification of T-boxes. Proceedings of the 21st International Workshop on Description Logic's (DL 2008), Dresden, Germany, May 13-16, 2008.

[7] Markus Fronk, Jens Lemcke.: Expressing Semantic Web Service Behavior using Description Logic's, 2006.

[8] Alaa Qassim, Al-Namiy.: Algorithm to Match Ontology's on the Semantic Web. (IJACSA) International Journal of Advanced Computer Science and Applications, Vol. 4, No.3, 2013.

[9] Andreas Steigmiller, Thorsten Liebig, Birte Glimm.: Konclude: System description. Web Semantics: Science, Services and Agents on the World Wide Web 2728, 2014. 